Marquette University

e-Publications@Marquette

College of Nursing Faculty Research and

Publications

Nursing, College of

$1-1-2017$

Effect of Sequence of Simulated and Clinical Practicum Learning Experiences on Clinical Competency of Nursing Students

Jamie Hansen

Marquette University

Marilyn Bratt

Marquette University, marilyn.bratt@marquette.edu

Accepted version. Nurse Educator, Vol 42, No. 5 (September/October 2017): 231-235. DOI. (C) 2017

Wolters Kluwer Health, Inc. Used with permission. 


\title{
Effect of Sequence of Simulated and Clinical Practicum Learning Experiences on Clinical Competency of Nursing Students
}

\author{
Jamie Hansen \\ Carroll University, \\ Waukesha, WI \\ Marilyn Bratt \\ College of Nursing, Milwaukee University \\ Milwaukee, WI
}

\begin{abstract}
Two different sequences of blocks of simulated and clinical practicum learning experiences compared the clinical competency development of nursing students using a randomized crossover design. Competency was measured 3 times: after each block of simulated and clinical experiences and after a final simulated experience. No significant differences in competency scores between the 2 groups across the 3 time points were found. Using alternative models of clinical and simulation learning may help address barriers to the delivery of clinical education faced by schools of nursing.
\end{abstract}

Nurse Educator, Vol 42, No. 5 (September/October 2017): pg. 231-235. DOI. This article is @ Wolters Kluwer Health, Inc. and permission has been granted for this version to appear in e-Publications@Marquette. Wolters Kluwer Health, Inc. does not grant permission for this article to be further copied/distributed or hosted elsewhere without the express permission from Wolters Kluwer Health, Inc. 
NOT THE PUBLISHED VERSION; this is the author's final, peer-reviewed manuscript. The published version may be accessed by following the link in the citation at the bottom of the page.

Keywords: clinical competency, clinical learning experiences, clinical practicum, nursing students, patient simulation

Providing quality learning experiences that foster the development of clinical competency in nursing students before entry into practice is a critical objective of all nursing educational programs. Traditionally, the model for practice-based learning in nursing involves faculty or preceptor supervision of students who are providing direct patient care in a hospital or other clinical settings $\frac{1,2}{}$ and can collectively be referred to as clinical learning experiences (CLEs). However, schools of nursing have increasingly faced barriers to provide clinical education in this manner because of factors such as increasing student enrollment numbers, a shortage of nursing faculty, increasing patient acuity, and decreasing clinical site availability. $\underline{2-4}$

Simulated learning experiences (SLEs) as a substitute for a portion of traditional CLE have gained interest for the past decade, but nurse educators continue to seek evidence supporting this practice. The National Council for State Boards of Nursing (NCSBN) recently conducted the National Simulation Study to explore student outcomes when CLEs were replaced with SLEs for $25 \%$ or $50 \%$ of the time allotted to clinical learning. $\frac{2,5}{2}$ Results of the study revealed no difference in student outcomes when substituting up to $50 \%$ of CLEs with SLEs. On the basis of these results, the NCSBN has challenged state boards of nursing to develop specific guidelines regarding the use of simulation in prelicensure nursing programs. 6 However, there is insufficient research on which to develop evidence-based practices to create new models of clinical education delivery that incorporate simulation as a component. In particular, because the NCSBN National Simulation Study did not study the influence of the sequence of CLEs and SLEs, this warrants further examination.

Research in human learning suggests that the sequence of learning activities may have an effect on student outcomes. $\frac{7.8}{}$ This is based on the principles that knowledge is built on previous learning, and the possession of appropriate background knowledge is essential for success in new learning situations. $\underline{7}$ A recent report indicated that students felt better prepared for the hospital setting if they had SLEs before their CLEs. ${ }^{9}$ As a result, this has led schools of nursing to place SLEs before CLEs to allow greater gains and transfer of knowledge to the clinical setting compared with the placement of SLEs after traditional CLEs.

Nurse Educator, Vol 42, No. 5 (September/October 2017): pg. 231-235. DOI. This article is @ Wolters Kluwer Health, Inc. and permission has been granted for this version to appear in e-Publications@Marquette. Wolters Kluwer Health, Inc. does not grant permission for this article to be further copied/distributed or hosted elsewhere without the express permission from Wolters Kluwer Health, Inc. 
To date, few studies in nursing address student outcomes and the development of competency using different sequences of CLEs and SLEs. $\underline{10-}$ 13 Most of these studies have examined students' performance outcomes and clinical competencies after their intermittent participation in SLEs during a semester rather than large blocks within a semester. Providing blocks of SLEs and CLEs has the potential to increase student enrollment and decrease the number of clinical units needed in a given semester by up to $50 \%$. ${ }^{1}$ However, it is unknown whether students' development of clinical competency is equivalent when a block of SLEs precedes or follows CLEs. In addition, when using the NLN/J effries Simulation Framework, it is suggested that researchers consider the impact of student demographic factors such as age on simulation-based competency outcomes. 4 Therefore, this study explored students' clinical competency outcomes following a unique model of clinical education delivery using 2 different sequences of blocks of SLEs and CLEs during students' first medicalsurgical nursing practicum rotation. The specific research questions were: (1) Does the sequence of blocks of SLEs and CLEs affect the clinical competency development of nursing students? and (2) Does the age of the student affect clinical competency development in the 2 different blocked sequences of SLES and CLES?

\section{Method}

\section{Design}

Using a randomized crossover design, this study was conducted at a large midwestern school of nursing and at various clinical units in metropolitan hospitals. Before the start of the semester, students enrolled in their first medical-surgical nursing practicum course were randomly assigned by the course coordinator to 1 of 2 sequences: SLE during the course of a 7-week period followed by CLE for 7 weeks (group S-C) or CLE for 7 weeks followed by SLE during the course of a 7-week period (group C-S). Students attended each block of SLE and CLE in the same group of 7 to 8 students.

\section{CLE and SLE}

For the CLE, students had two 8-hour clinical days per week in which they provided direct care for 1 patient under the supervision of a

Nurse Educator, Vol 42, No. 5 (September/October 2017): pg. 231-235. DOI. This article is (C Wolters Kluwer Health, Inc. and permission has been granted for this version to appear in e-Publications@Marquette. Wolters Kluwer Health, Inc. does not grant permission for this article to be further copied/distributed or hosted elsewhere without the express permission from Wolters Kluwer Health, Inc. 
faculty member. During this experience, students planned, implemented, and evaluated nursing care and participated in a postconference discussion. For the SLE, spread over a 7-week period, students participated in 3 high-fidelity simulation days, each lasting 4 hours, and 1 medium-fidelity virtual simulation occurring between the first and second high-fidelity simulation days. Each high-fidelity simulation followed the NLN/J effries framework for simulation ${ }^{2}$ and included 4 vignettes for each simulation day on topics including pain management, heart failure, and chronic obstructive pulmonary disease/pneumonia. For the medium-fidelity virtual simulation, students completed independently a computergenerated program on the topic of diabetes mellitus that included preselected debriefing questions. Simulations were run by instructors who received training before the start of the study along with a step-by-step manual with instructions to ensure all SLE and debriefing sessions were run as similarly as possible. To standardize the debriefing method, a tool was developed using the Sim TRACT model. $\underline{14}$

For each high-fidelity simulation day, students were to complete assigned prework including text readings, a quiz focused on the simulation topic, a tentative plan of care, and a review of the specific simulation scenario objectives, associated patient charts, laboratory results, and medication administration record. A prebriefing session and orientation to the high-fidelity simulation room and manikin were conducted before the start of each simulation. Each simulation vignette included 2 active student roles, the primary RN, and the primary RN's preceptor. During vignettes, students performed patient assessments and required nursing interventions followed by a debriefing session conducted by the simulation instructor. The remaining students in the clinical group observed vignettes via a live video feed in a debriefing room with their instructor and took notes to provide feedback to the active simulation student participants or identify any areas needing clarification during the debriefing session. For the medium-fidelity simulation, students worked through the assigned scenario that required application of the nursing process to address the simulated patient's evolving problems. To guide students during this learning exercise, the online program incorporated prebriefing and postscenario debriefing. After completion of their SLE and CLE sequences, students were evaluated during a final high-fidelity simulation vignette approximately 5 weeks after the completion of the semester but before the start of the subsequent semester. A unique type II diabetes mellitus vignette using the same format and with a similar level of complexity as

Nurse Educator, Vol 42, No. 5 (September/October 2017): pg. 231-235. DOI. This article is (C) Wolters Kluwer Health, Inc. and permission has been granted for this version to appear in e-Publications@Marquette. Wolters Kluwer Health, Inc. does not grant permission for this article to be further copied/distributed or hosted elsewhere without the express permission from Wolters Kluwer Health, Inc. 
the previous high-fidelity simulation vignettes was used for the final evaluation time point.

\section{Sample}

Sample size calculation was conducted a priori power using the software program G* Power version 3.0.10. The estimated required sample size for a between-group repeated-measures analysis of variance (RMANOVA) with 2 groups, 3 measurement time points, an $a$ level of .05, a minimal statistical power of 0.8 , and what is considered a small to medium effect size, $d=0.35 \underline{15}$ was 46 students ( 23 per group). This effect size was chosen based on those reported in the NCSBN study using the Creighton

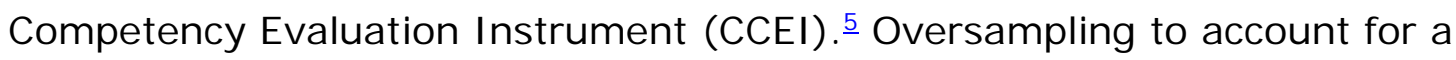
potential $30 \%$ dropout rate brought the target sample to 60 students.

All students enrolled in the practicum course were invited to be in the study. A convenience sample of nursing students was recruited using the following inclusion criteria: (1) at least 18 years old, (2) enrolled in their first medical-surgical nursing course, and (3) enrolled in the associated medical-surgical nursing theory course.

\section{Data Collection and Measurement}

After university institutional review board approval and obtaining participating students' consent, demographic information was collected. Evaluation of students' clinical competency was measured using the CCEI 3 times: (1) during a designated simulation vignette at the end of students' SLE rotation, (2) during a preselected clinical day involving a single patient encounter occurring in the final week of students' CLE rotation, and (3) after completion of the semester during a follow-up simulation vignette. The ratio of simulation to clinical hours for the semester was 1:4. For the study, students were evaluated when they were assigned to the primary $\mathrm{RN}$ role in a simulation vignette.

The CCEI is a 23-item tool with 4 subscales: assessment ( 3 items), communication ( 5 items), clinical judgment ( 9 items), and patient safety ( 6 items). .16 To score the tool, each item is assigned a 0 or 1 depending if a specific behavior is demonstrated (scored as 1) or not demonstrated/not applicable (scored as 0 ). Therefore, the total score on the tool can range 
from 0 to 23. Previous studies have demonstrated acceptable reliability estimates with Cronbach's $a$ ranging from .97 to $.98 . \frac{16,17}{1 n}$ this study, Cronbach's $a$ for the total scale was .95, and those for the subscales ranged from .94 to .99.

To ensure interrater reliability of the instrument for this study, 16 instructors received training on the use of the CCEI tool before the start of data collection. During the training session, each instructor viewed a series of videos provided by the tool developers that gave an orientation to the tool and discussion of how to properly score students' expected behaviors for each item on the instrument. To establish interrater reliability, instructors then viewed and independently scored an archived video scenario using the CCEI. The researcher, who was deemed an expert rater, previously scored the same archived video to determine the discrepancy of ratings among instructors. If scores differed by more than 4 points $(<80 \%$ consensus), additional instructor training was to be conducted by the researcher; however, no additional training was needed. Interrater reliability of the CCEI in this study demonstrated an overall percentage agreement with the researcher of $92 \%$. To account for the amount of agreement expected due to chance, $\mathrm{k}$ statistics were also calculated $\underline{18,19}$ and suggested moderate to almost perfect agreement $(\kappa=0.481-1)$.

\section{Data Analysis}

Descriptive and inferential statistical methods were used to analyze the data using SPSS version 23.0. To ensure that the 2 groups were equivalent at baseline, pertinent demographic variables were compared using $\mathrm{X}^{2}$ analysis for categorical variables and independent $\mathrm{t}$ tests for continuous variables. To determine whether clinical competency using the CCEI differed for the 3 measurement time points within and between the 2 groups, RM-ANOVA was calculated. To establish statistical significance, an $a$ level of .05 was used. Significant main effects of group, time, or interaction were explored further through post hoc comparison using simple main effects analysis. All analyses included only those students who had complete data across all 3 measurement time points.

\section{Results}

\section{Sample Demographic Characteristics}

Nurse Educator, Vol 42, No. 5 (September/October 2017): pg. 231-235. DOI. This article is (C) Wolters Kluwer Health, Inc. and permission has been granted for this version to appear in e-Publications@Marquette. Wolters Kluwer Health, Inc. does not grant permission for this article to be further copied/distributed or hosted elsewhere without the express permission from Wolters Kluwer Health, Inc. 
NOT THE PUBLISHED VERSION; this is the author's final, peer-reviewed manuscript. The published version may be accessed by following the link in the citation at the bottom of the page.

Of the 120 students initially invited to participate in the study, 71 enrolled for a $59 \%$ response rate. Of the 71 originally enrolled, 48 participated in all 3 data collection time points, for a $67.6 \%$ retention rate. The final sample consisted primarily of white women with a mean (SD) age of 22.2 (3) years, as presented in the Table, Supplemental Digital Content 1, http://links.Iww.com/NE/A328. No statistically significant differences between groups were identified for any of the variables describing the sample characteristics.

\section{Differences Between Groups: Group S-C Versus Group C-S}

The primary aim of this study was to determine whether the sequence of blocks of SLEs and CLEs impacted clinical competency development in nursing students participating in their first medical-surgical practicum course. Results showed that there were no significant differences in CCEI total $\left(F_{1,46}=0.05, P=.811\right)$ or subscale scores between the 2 groups across the 3 data collection points. Consequently, there was no significant effect on clinical competency based on the sequence participants were assigned to.

\section{Differences Within Groups}

As illustrated in the Table, there was a significant time-by-group interaction for CCEI total scores. Simple main effects analysis revealed that both groups had significantly higher scores after the CLE component of the sequence, with group S-C demonstrating significantly higher CCEI total scores at time 2 compared with times 1 and 3 and group C-S demonstrating significantly higher total CCEI scores at time 1 compared with time 3. Of note, there were significant time-by-group interactions among the CCEI subscales. Mauchly's test indicated that the assumption of sphericity for the patient safety subscale was violated, $\mathrm{P}=.009$; therefore, the degrees of freedom were corrected using Huynh-Feldt $(\varepsilon=0.925)$. Simple main effects analyses revealed that group S-C demonstrated significantly higher scores for the assessment and patient safety subscales at time 2 after CLE compared with times 1 and 3 and significantly higher scores for the communication and clinical judgment subscales at time 2 after CLE compared with time 1. Group C-S demonstrated significantly higher assessment subscale scores at time 1 after CLE compared with time 2 , significantly higher clinical judgment subscale scores at time 3 
compared with time 2, and significantly higher patient safety subscale scores at time 1 after CLE compared with times 2 and 3.

\begin{tabular}{|c|c|c|c|c|}
\hline & \multirow[b]{2}{*}{ Time 1, Mean (SD) } & \multirow[b]{2}{*}{ Time 2, Mean (SD) } & \multirow[b]{2}{*}{ Time 3, Mean (SD) } & $P$ of Differences Between Periods \\
\hline & & & & Times 1-2 (a)/Times 1-3 (b)/Times 2-3 (c) \\
\hline \multicolumn{5}{|l|}{ Group S-C $(\mathrm{N}=22)$} \\
\hline Total score & $19.05(3.3)$ & $21.73(3.1)$ & $19.14(2.7)$ & $.001(\mathrm{a}) / .005(\mathrm{c})$ \\
\hline Assessment & $2.09(0.75)$ & $2.64(0.90)$ & $1.86(0.71)$ & $.014(\mathrm{a}) / .003(\mathrm{c})$ \\
\hline Communication & $4.18(0.91)$ & $4.73(0.70)$ & $4.55(0.67)$ & .033 (a) \\
\hline Clinical judgment & $8.05(1.2)$ & $8.73(0.77)$ & $8.36(1.2)$ & .046 (a) \\
\hline Patient safety & $4.73(1.3)$ & $5.64(1.0)$ & $4.36(1.4)$ & $.001(\mathrm{a}) / .003(\mathrm{c})$ \\
\hline \multicolumn{5}{|l|}{ Group C-S $(\mathrm{N}=26)$} \\
\hline Total score & $21.12(3.3)$ & $20.01(2.8)$ & $19.15(2.6)$ & .018 (b) \\
\hline Assessment & $2.50(0.95)$ & $2.04(0.92)$ & $2.12(0.82)$ & .020 (a) \\
\hline Communication & $4.69(0.74)$ & $4.77(0.71)$ & $4.73(0.45)$ & NS \\
\hline Clinical judgment & $8.19(1.7)$ & $8.15(0.88)$ & $8.69(0.68)$ & $.010(\mathrm{c})$ \\
\hline Patient safety & $5.73(0.53)$ & $5.08(1.2)$ & $3.62(1.6)$ & $.002(\mathrm{a}$ and $\mathrm{c}) / .001(\mathrm{~b})$ \\
\hline
\end{tabular}

\section{Age, Sequence, and Clinical Competency Development}

The secondary aim of the study was to determine whether the age of the learner affected clinical competency development in the different blocked sequences of SLEs and CLEs. There was no significant effect of age and group on CCEI total scores $\left(F_{2,88}=0.800, P=.452\right)$ and subscale scores.

Nurse Educator, Vol 42, No. 5 (September/October 2017): pg. 231-235. DOI. This article is (C) Wolters Kluwer Health, Inc. and permission has been granted for this version to appear in e-Publications@Marquette. Wolters Kluwer Health, Inc. does not grant permission for this article to be further copied/distributed or hosted elsewhere without the express permission from Wolters Kluwer Health, Inc. 


\section{Discussion}

Results of this study provide evidence regarding the effects of blocked sequences of SLEs and CLEs on clinical competency development. In this study, students' age did not have a significant influence on CCEI total scores or subscales. This is similar to previous reports in the simulation literature regarding age and simulation outcomes.,20 These insignificant findings may have been due to the lack of age variability in the students participating in the study. Despite these insignificant findings, student demographic factors should continue to be investigated in studies exploring simulation-based competency outcomes particularly using a sample compromised of a more diverse student population, as suggested by Ironside et al. 4

Findings revealed that there were no between-group differences in the CCEI total or subscale scores noted during the study period, suggesting that the sequence of SLEs and CLEs did not impact participants' CCEI scores over time. Of interest, there were several notable withingroup differences for this sample. Regardless of group assignment, students had higher total CCEI scores after the CLE portion of the sequence. Although the CCEI was initially developed for use exclusively in the simulation environment, the current version of the tool has been reported to be reliable and valid in both the clinical and simulation environments. $\frac{16}{}$ However, no studies to date have compared the faculty ratings of students in the clinical and simulation environments using the tool. Therefore, it is possible that higher scores after students' CLEs are a function of the environment in which they were evaluated. Further study of the CCEI tool is warranted to determine whether student scores in the clinical environment are significantly different than those obtained in the simulation environment.

Further examination of clinical competency through an analysis of the CCEI subscales revealed significant within-group changes over time. Clinical judgment subscale scores were significantly higher for each group post-SLE. Previous reports have suggested that simulation contributes to

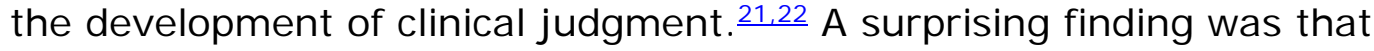
students scored the lowest in the demonstration of patient safety subscale behaviors during the final simulation vignette regardless of group assignment. This is contrary to other studies that have found significant 
improvements in patient safety competency scores after simulation. $\frac{4,23}{}$ Decreased demonstration of these safety behaviors may have been attributed to an approximately 5-week gap between the second and final measurement points, during which students were between semesters and not attending classes. This gap could have ultimately impacted the retention of key behaviors that are included in this subscale such as medication administration, correct performance of procedures, and use of patient identifiers. Previous studies have reported significant decline in skills performance using high-fidelity simulation after a lapse of time between evaluations. $\frac{24,25}{}$ On the basis of these findings, a further study that examines the retention of procedural knowledge comparing the alternative models of clinical education delivery is needed.

\section{Limitations}

Because this study explored the influence of 2 different sequences of SLEs and CLEs on clinical competency in only 1 practicum course at 1 university, the generalizability of the results is limited to other courses, curricular levels, or nursing programs. It is also possible that the 2 groups were not equivalent because no pretest measure of clinical competency was obtained.

\section{I mplications for Nursing Education}

This study provides evidence that participation in a block of SLEs preceding or after a block of CLEs may produce similar student outcomes regardless of the sequence of these learning experiences. This unique model of clinical education delivery in nursing programs may aid in addressing the barriers faced by nurse educators such as lack of clinical site availability and increased student enrolment. There is a need for additional appraisal of the CCEI tool comparing its reliability for use in the clinical and simulation settings to determine whether differences in faculty evaluation of student performance vary depending on the environment. Continued assessment of student outcomes using alternative formats of simulation and clinical hours, in additional courses, and for longer periods is necessary before nurse educators can determine the optimal clinical education delivery model for prelicensure nursing programs.

\section{References}

Nurse Educator, Vol 42, No. 5 (September/October 2017): pg. 231-235. DOI. This article is (C Wolters Kluwer Health, Inc. and permission has been granted for this version to appear in e-Publications@Marquette. Wolters Kluwer Health, Inc. does not grant permission for this article to be further copied/distributed or hosted elsewhere without the express permission from Wolters Kluwer Health, Inc. 
NOT THE PUBLISHED VERSION; this is the author's final, peer-reviewed manuscript. The published version may be accessed by following the link in the citation at the bottom of the page.

${ }^{1}$ Richardson H, Goldsamt LA, Simmons J, Gilmartin M, Jeffries PR. Increasing faculty capacity: findings from an evaluation of simulation clinical teaching. Nurs Educ Perspect. 2014; 35(5): 308-314.

2J effries PR. Simulation in Nursing Education: From Conceptualization to Evaluation. 2nd ed. New York, NY: National League for Nursing; 2012.

${ }^{3}$ Bensfield LA, Olech MJ, Horsley TL. Simulation for high-stakes evaluation in nursing. Nurse Educ. 2012;37(2):71-74.

${ }^{4}$ Ironside $\mathrm{PM}$, J effries PR, Martin A. Fostering patient safety competencies using multiple-patient simulation experiences. Nurs Outlook. 2009;57(6):332337.

${ }^{5}$ Hayden JK, Smiley RA, Alexander M, Kardong-Edgren S, Jeffries PR. The NCSBN national simulation study: a longitudinal, randomized, controlled study replacing clinical hours with simulation in prelicensure nursing education. J Nurs Regul. 2014; 5(2): C1-S64.

${ }^{6}$ Alexander M, Durham CF, Hooper JI, et al. NCSBN simulation guidelines for prelicensure nursing programs. J Nurs Regul. 2015;6(3):39-42.

${ }^{7}$ Ritter FE, Nerb J, Lehtinen E, O'Shea TM. In Order to Learn: How the Sequence of Topics Influences Learning. Oxford, England: Oxford University Press; 2007.

${ }^{8}$ Rohrer $\mathrm{D}$, Pashler $\mathrm{H}$. Recent research on human learning challenges conventional instructional strategies. Educ Res. 2010;39(5):406-412.

${ }^{9}$ Woda AA, Gruenke T, Alt-Gehrman P, Hansen J. Nursing student perceptions regarding simulation experience sequencing. J Nurs Educ. 2016; 55(9): 528-532.

${ }^{10}$ Curl ED, Smith S, Ann Chisholm L, McGee LA, Das K. Effectiveness of integrated simulation and clinical experiences compared to traditional clinical experiences for nursing students. Nurs Educ Perspect. 2016;37(2):72- 77.

${ }^{11}$ Meyer MN, Connors H, Hou Q, Gajewski B. The effect of simulation on clinical performance. Simul Healthc. 2011;6(5):269-277.

${ }^{12}$ Schlairet MC, Fenster MJ. Dose and sequence of simulation and direct care experiences among beginning nursing students: a pilot study. J Nurs Educ. 2012; 51(12): 668-675.

${ }^{13}$ Schlairet MC, Pollock JW. Equivalence testing of traditional and simulated clinical experiences: undergraduate nursing students' knowledge acquisition. J Nurs Educ. 2010;49(1):43-47.

${ }^{14}$ Gum L, Greenhill J, Dix K. Sim TRACT: a reflective conceptual framework for simulation debriefing. J Transform Educ. 2011;9(1):21-41.

${ }^{15}$ Cohen J . Statistical Power Analysis for the Behavioral Sciences. 2nd ed. Hillside, NJ : Erlbaum; 1988.

${ }^{16}$ Hayden J, Keegan M, Kardong-Edgren S, Smiley RA. Reliability and validity testing of the Creighton competency evaluation instrument for use in the NCSBN national simulation study. Nurs Educ Perspect. 2014;35(4):244252.

Nurse Educator, Vol 42, No. 5 (September/October 2017): pg. 231-235. DOI. This article is @ Wolters Kluwer Health, Inc. and permission has been granted for this version to appear in e-Publications@Marquette. Wolters Kluwer Health, Inc. does not grant permission for this article to be further copied/distributed or hosted elsewhere without the express permission from Wolters Kluwer Health, Inc. 
${ }^{17}$ Adamson KA, Parsons ME, Hawkins K, Manz JA, Todd M, Hercinger M. Reliability and internal consistency findings from the C-SEI. J Nurs Educ. 2011; 50(10): 583-586.

${ }^{18}$ Landis JR, Koch GG. The measurement of observer agreement for categorical data. Biometrics. 1977;33(1): 159-174.

${ }^{19}$ Waltz CF, Strickland OL, Lenz ER. Measurement in Nursing and Health Research. New York, NY: Springer; 2010.

${ }^{20}$ Lasater K. The Impact of High Fidelity Simulation on the Development of Clinical Judgment in Nursing Students: An Exploratory Study [dissertation]. 2005. Available at http://search.proquest. com/docview/305452825?accountid=100. Accessed January 12, 2017.

${ }^{21}$ Fisher D, King L. An integrative literature review of preparing nursing students through simulation to recognize and respond to the deteriorating patient. J Adv Nurs. 2013;69(11):2375-2388.

${ }^{22}$ Lasater K. Clinical judgment development: using simulation to create an assessment rubric. J Nurs Educ. 2007;46(11):496-503.

${ }^{23}$ Sears K, Goldsworthy S, Goodman WM. The relationship between simulation in nursing education and medication safety. J Nurs Educ. 2010;49(1):52-55.

${ }^{24}$ Aqel AA, Ahmad MM. High-fidelity simulation effects on CPR knowledge, skills, and retention in nursing students. Worldviews Evid Based Nurs.

2014; 11(6):394-400.

${ }^{25}$ Ross JG. Simulation and psychomotor skill acquisition: a review of the literature. Clin Simul Nurs. 2012; 8(9): e429-e435.

\section{Supplemental Digital Content}

Table. Demographic Characteristics

\begin{tabular}{llll}
\hline $\begin{array}{l}\text { Characteristic } \\
\text { 26) }\end{array}$ & All Participants $(\mathrm{N}=48)$ & Group S-C $(\mathrm{n}=22)$ & Group C-S ( $=$ \\
\hline Age: Mean (SD) & $22.2(3.0)$ & $21.9(1.9)$ & $22.4(3.7)$ \\
Female: \% (n) & $79.2(3)$ & $72.7(16)$ & $84.6(22)$ \\
Caucasian: \% (n) & $83.3(40)$ & $77.3(17)$ & $88.5(23)$ \\
Prior Degree: \% (n) & $47.9(23)$ & $50(11)$ & $46.1(12)$ \\
Work in Healthcare: \% (n) & $27(13)$ & $22.7(5)$ & $30.8(8)$ \\
\hline
\end{tabular}

Nurse Educator, Vol 42, No. 5 (September/October 2017): pg. 231-235. DOI. This article is (C Wolters Kluwer Health, Inc. and permission has been granted for this version to appear in e-Publications@Marquette. Wolters Kluwer Health, Inc. does not grant permission for this article to be further copied/distributed or hosted elsewhere without the express permission from Wolters Kluwer Health, Inc. 